\title{
Phenotypic characterization of patient dengue virus isolates in BALB/c mice differentiates dengue fever and dengue hemorrhagic fever from dengue shock syndrome
}

Anne Tuiskunen ${ }^{1,2,3^{*}}$, Maria Wahlström², Jakob Bergström², Philippe Buchy ${ }^{4}$, Isabelle Leparc-Goffart ${ }^{3}$ and Åke Lundkvist ${ }^{1,2}$

\begin{abstract}
Background: Dengue virus (DENV) infection is the most common arthropod-borne viral disease in man and there are approximately 100 million infections annually. Despite the global burden of DENV infections many important questions regarding DENV pathogenesis remain unaddressed due to the lack of appropriate animal models of infection and disease. A major problem is the fact that no non-human species naturally develop disease similar to human dengue fever (DF) or dengue hemorrhagic fever (DHF) and dengue shock syndrome (DSS). Apart from other risk factors for severe dengue such as host genetics and secondary infection with a heterologous DENV, virus virulence is a risk factor that is not well characterized.
\end{abstract}

Results: Three clinical DENV-1 isolates from Cambodian patients experiencing the various forms of dengue disease (DF, DHF, and DSS) were inoculated in BALB/c mice at three different concentrations. The DENV-1 isolates had different organ and cell tropism and replication kinetics. The DENV-1 isolate from a DSS patient infected the largest number of mice and was primarily neurotropic. In contrast, the DENV-1 isolates from milder clinical dengue cases infected predominantly lungs and liver, and to a lesser extent brain. In addition, infection with the DENV isolate derived from a DSS patient persisted for more than two weeks in a majority of mice compared to the other DENV1 isolates that peaked during the first week.

Conclusions: These results confirm the in vitro findings of the same DENV-1 isolates, that showed that the isolate derived from a DSS patient can be distinguished based on phenotypic characteristics that differ from the isolates derived from a DF and DHF case [1]. We observed in this study that the DSS virus isolate persist longer in vivo with extensive neuroinvasion in contrast to the other DENV-1 isolates originating in milder human cases. Genomic characterization of the three clinical isolates identified six amino acid substitutions unique for the DSS isolates that were located both in structural genes (M and E) and in non-structural genes (NS1, NS3, and NS5). The characterization of these clinically distinct DENV-1 isolates highlight that DENVs within the same genotype may have different in vivo phenotypes.

Highlights: • Clinical DENV-1 isolates have different organ tropism in BALB/C mice.

- The isolate from a DSS patient is primarily neurotropic compared to the other isolates.

- The DENV-1 isolates have different in vivo replication kinetics.

- The isolate from a DSS patient persists longer compared to the other isolates.

- These phenotypic differences confirm our earlier in vitro findings with the same DENV-1 isolates. Thus, DENVs within the same serotype and genotype may differ enough to affect clinical conditions in vivo.

\footnotetext{
* Correspondence: anne.tuiskunen@ki.se

'Department of Microbiology, Tumor and Cell Biology, Karolinska Institutet,

Stockholm, Sweden

Full list of author information is available at the end of the article
} 


\section{Background}

The dengue viruses (DENV) belong to the genus flavivirus of the Flaviviridae family, and consist of four (1-4) antigenically related, but clearly distinct viruses (serotypes). The DENV particle is enveloped, and has a single-stranded positive-sense RNA genome of approximately $11 \mathrm{~kb}$ that resembles a messenger RNA with a cap on the 5' end but no poly(A) tail at the 3' end. The RNA genome encodes a 3411 long precursor polyprotein that contains three structural proteins $(C$, prM, and E), and seven nonstructural proteins (NS1, NS2A, NS2B, NS3, NS4A, NS4B, and NS5). The open reading frame is flanked by two nontranslated regions (5' and 3' NTR) of approximately 95-135 and 114-650 nucleotides, respectively, that have characteristic secondary structures that are required for efficient translation and replication $[2,3]$.

The DENVs are endemic in tropical and subtropical areas and more than one hundred million people get infected annually. Infection can be either asymptomatic, or cause an acute febrile illness that is characterized by fever, headache, retro-orbital pain, arthralgia, and myalgia. This condition can progress into dengue haemorrhagic fever (DHF), with cardinal signs such as increased vascular permeability, thrombocytopenia, focal or generalized haemorrhages. DF may progress into the life-threatening state of dengue shock syndrome (DSS) [4]. In the recent WHO guidelines, for case management purposes, DHF and DSS cases are now grouped together as "severe dengue" (group C) [5].

Uncontrolled urbanization and globalization have resulted in the geographic spread of the DENV-transmitting mosquitoes Aedes aegypti and A. albopictus, cocirculation of different DENV serotypes, and increased frequency of dengue epidemics [6]. There has been a severe increase of DHF/DSS in many endemic regions, emphasizing the urgent need of an effective vaccine.

Despite the global burden of DENV infections many important questions regarding DENV pathogenesis remain unaddressed due to the lack of appropriate animal models of infection and disease. A major problem is the fact that no non-human species naturally develop disease similar to human DF or DHF/DSS. Epidemiological, clinical, and laboratory findings indicate that both genetic differences in the virus and the host immune response contribute to the occurrence and severity of disease [7].

In the search for better animal models for dengue, several approaches to investigate infection of DENV in mouse have been proposed. One of two major strategies have been to modulate the mouse to render it more susceptible to DENV infection, e.g. SCID mice transplanted with cultured, DENV-susceptible tumour cells [8-10]. The other major alternative has been to modify the DENV in order to make it more infection-competent in the murine host, e.g. mouse-neuroadapted DENV [11-14], or virus serially passaged in mouse $[15,16]$. The relevance of these models to human pathogenesis remains unknown.

The immunocompetent BALB/c mouse is susceptible to DENV infection and has been used extensively to study various aspects of DENV infection pathogenesis, despite the lack of clinical symptoms [17-20]. Mouseadapted DENV strains increase the susceptibility to DENV infection and elicit clinical symptoms in BALB/c mice $[11,21]$. The relevance of these observations to infection with wild-type DENV strains, however, should be interpreted with caution due to the altered genotype and phenotype of the passaged virus.

We have previously reported that DENV-1 isolates only passaged once in C6/36 cells (passage 1), derived from patients presenting the clinically distinct forms of dengue (DF, DHF, and DSS), can be distinguished in vitro based on different replication kinetics in mammalian Vero cells and apoptosis in C6/36. The DSS-derived isolate (accession number: FJ639694) had a unique phenotype compared to the DF- and DHF-derived isolates (accession numbers: HQ624983 and HQ624984, respectively) and genomic comparison revealed six amino acid substitutions unique for the DSS isolate that were located in the structural viral $\mathrm{M}$ and $\mathrm{E}$ genes that constitute the extracellular mature virus particle, and in the NS1, NS3, and NS5 genes. The E protein mediates host cell receptor binding, viral entry, and is a major target for humoral immunity [22,23]. The role of NS1 in virus replication is not known, but is thought to be involved in facilitation of viral infection and in the pathogenesis of DENV infection $[24,25]$. The NS3 acts together with its cofactor NS2B as the viral serine protease needed for DENV precursor polyprotein-processing [3,26-28]. The NS5 protein has a dual enzymatic activity with an RNA dependent RNA polymerase activity in its C-terminal domain, and a methyltransferase activity at its N-terminal end. Hence, these six mutations could ultimately affect antibody response, host immune pathways, and virus replication.

The primary aim of the present study was to investigate whether there is a phenotype difference in vivo 
between the DENV-1 isolates similar to the in vitro findings, by studying difference in infectivity in BALB/c mice and to investigate the cell and organ tropism. The second aim was to determine if the inoculated virus concentration and the effects of the infection correlated, primarily by measuring the levels of inflammatory cytokines induced by the infection.

\section{Results}

\section{DENV infection and Organ Tropism}

All mice had detectable levels of DENV specific IgG antibodies at day 15 post-infection (p.i.), confirming the intravenous route to be appropriate choice in order to successfully infect all mice. In total $54.3 \%$ of the DENV1 infected mice had detectable levels of viral RNA; $48.2 \%$ of the mice infected with the DF isolate; $55.6 \%$ of the DHF isolate infected mice; and $59.3 \%$ of the DSS isolate infected mice (table 1). All mice survived the infection without presenting any clinical signs but pathological lesions such as haemorrhage could be detected, most notably in brain, liver, lung tissue, and to some extent in spleen.

Haemorrhage in lungs was most pronounced in the DF-infected mice, whereas the DHF-infected mice experienced haemorrhage in lungs, liver and to some extent in brain. The DSS isolate seemed to have some neurotropism as the DSS-infected mice had extensive bleedings in the brain, whereas the peripheral organs showed only minor signs of haemorrhage. None of the NC mice exhibited any clinical signs or tissue pathology.

The three DENV-1 isolates showed different tropism; the two DENVs obtained from a DF and DHF case infected primarily lungs, spleen, and liver, whereas the DENV from a DSS patient infected lungs, spleen, and exhibited a strong tropism for brain tissue $(p=0.001)$. The results are summarized in table 1 . The effects of infection in lung tissue showed a positive correlation to inoculated virus concentration and time-point, with a peak of infection at day 6 p.i. $(p=0.003)$ combined with the highest titer $1.5 \times 10^{6} \mathrm{PFU} / \mathrm{mL}$ in the inoclum $(p<$ 0.001) (table 2).

\section{Risk of Infection and Kinetics of Infection}

The DENV-1 isolates exhibited different risks for infection that depended both on virus concentration in the inoculum $(p=0.012)$ and on time-point p.i. $(p=0.014)$ (Figure 1). The effects of inoculated dose of infectious virus showed a positive correlation to the total number of infected mice for each virus isolate, and infection peaked on day 6 p.i. for all three viruses.

Kinetics of infection differed between the three DENV-1 isolates. Viral RNA in the DF and DHF inoculated mice was predominantly detected during the first week of infection, and declined during the second week. Six inoculated mice tested positive after inoculation with the DF, and DHF isolates, respectively, both on day 3 and 6 p.i. During the second week (day 15 p.i.), however, only 1 and 3 mice tested positive after inoculation with the DF- and DHF-viruses, respectively. The timecourse of infection differed in the DSS- infected mice that showed a delayed response to inoculation compared to the DF- and DHF-infected mice. Day 3 p.i. had the lowest number of DSS-infected mice (3 positive mice) whereas at day 6 p.i. 7 mice were infected, and 6 during

Table 1 Viral RNA was found in various organs depending on inoculated DENV-1 isolate (DF, DHF, or DSS), virus concentration in inoculum, and time-point p.i. In total $54.3 \%$ of the DENV-1 infected mice had detectable levels of viral RNA

\begin{tabular}{|c|c|c|c|c|c|c|c|c|c|c|c|c|c|c|c|c|c|c|c|c|c|c|c|c|c|c|c|}
\hline \multirow{3}{*}{$\frac{\frac{\text { DENV isolate }}{\text { Titer }(\mathrm{PFU} / \mathrm{mL})^{*}}}{\text { Day }(\text { p.i. })^{* *}}$} & \multicolumn{9}{|c|}{ DF } & \multicolumn{9}{|c|}{ DHF } & \multicolumn{9}{|c|}{ DSS } \\
\hline & \multicolumn{3}{|c|}{$1.5 \times 10^{4}$} & \multicolumn{3}{|c|}{$1.5 \times 10^{5}$} & \multicolumn{3}{|c|}{$1.5 \times 10^{6}$} & \multicolumn{3}{|c|}{$1.5 \times 10^{4}$} & \multicolumn{3}{|c|}{$1.5 \times 10^{5}$} & \multicolumn{3}{|c|}{$1.5 \times 10^{6}$} & \multicolumn{3}{|c|}{$1.5 \times 10^{4}$} & \multicolumn{3}{|c|}{$1.5 \times 10^{5}$} & \multicolumn{3}{|c|}{$1.5 \times 10^{6}$} \\
\hline & 3 & 6 & 15 & 3 & 61 & 15 & 3 & 6 & 15 & 3 & 6 & 15 & 3 & 6 & 15 & 3 & 6 & 15 & 3 & 6 & 15 & 3 & 6 & 15 & 3 & 6 & 15 \\
\hline Heart & - & - & - & - & - & - & - & - & - & - & - & - & - & - & - & - & - & - & - & - & - & - & - & - & - & - & - \\
\hline Lungs & - & - & - & 2 & 3 & - & 2 & 3 & 1 & - & - & - & - & 2 & - & 2 & 2 & 1 & - & - & - & - & 3 & 1 & 1 & 1 & 1 \\
\hline Spleen & 1 & - & - & - & - & - & 2 & - & - & 1 & - & - & - & - & 2 & 2 & 1 & - & - & 1 & - & - & 1 & 1 & 2 & - & - \\
\hline Brain & - & - & - & - & - & - & 1 & - & - & 1 & 1 & - & - & - & - & - & 3 & - & - & 1 & 2 & 1 & 3 & 2 & - & 2 & - \\
\hline Liver & - & - & - & - & - & - & - & - & - & - & - & - & 1 & 1 & - & 2 & - & - & - & - & - & - & - & - & - & - & - \\
\hline Kidneys & - & - & - & - & - & - & - & - & - & - & - & - & - & - & - & - & - & - & - & 1 & - & - & - & - & - & - & - \\
\hline Infected mice & 1 & - & - & 2 & 3 & - & 3 & 3 & 1 & 2 & 1 & - & 1 & 2 & 2 & 3 & 3 & 1 & - & 3 & 2 & 1 & 3 & 3 & 2 & 2 & 1 \\
\hline Infected mice per titer & & 1 & & & 5 & & & 7 & & & 3 & & & 5 & & & 7 & & & 4 & & & 7 & & & 5 & \\
\hline Infected mice per day p.i. & 6 & & & & 6 & & & & 1 & 6 & & & & 6 & & & & 3 & 3 & & & & 7 & & & & 6 \\
\hline$\Sigma$ infected mice per virus isolate & & & & & 13 & & & & & & & & & 15 & & & & & & & & & 16 & & & & \\
\hline$\%$ infected mice per virus isolate & & & & & $48.2 \%$ & & & & & & & & & $55.6 \%$ & & & & & & & & & $59.3 \%$ & & & & \\
\hline
\end{tabular}

* Titer of inoculum was significant $(p=0.012)$.

** Time-point post-infection was significant $(p=0.014)$. 
Table 2 Risk of infection in lungs

\begin{tabular}{cc}
\hline $\begin{array}{c}\text { Inoculated virus } \\
\text { (PFU/mL) }\end{array}$ & Risk of infection \\
\hline $1.5 \times 10^{4}$ & 0 \\
$1.5 \times 10^{5}$ & 0.41 \\
$1.5 \times 10^{6}$ & 0.52 \\
Days p.i. & Risk of infection \\
3 & 0.26 \\
6 & 0.52 \\
15 & 0.15 \\
\hline
\end{tabular}

Both inoculated DENV-1 concentration and time-point were significant $(p<$ 0.001 and $p=0.003$, respectively). The risk for infection in the lungs with the DF isolate was highest on day 6 p.i. and showed a positive correlation to inoculated virus.

the second week of infection (day 15 p.i.). Thus, the number of mice infected with the DF and DHF isolates peaked during day 3 and 6 p.i. in contrast to the number of DSS-infected mice that peaked during day 6 and 15 p.i (Figure 2).

Overall, the DSS isolate replicated in a majority of infected mice and for a longer period of time compared to the DENVs obtained from milder cases (Figure 2).

\section{Cytokine secretion in serum}

IFN $\gamma$ levels peaked during the first week of infection and most notably on day 3 p.i. that showed the highest levels of $\operatorname{IFN} \gamma(p=0.007)$. There was also a positive correlation to inoculated virus dose where the highest titer $\left(1.5 \times 10^{6} \mathrm{PFU} / \mathrm{mL}\right)$ elicited the strongest IFN $\gamma$ response $(p=0.002)$. A positive titer dependent correlation to IL-6 levels was observed in all three groups of DENV-1 inoculated mice $(p=0.028)$ (data not shown). There was a time and virus dependent difference regarding detected IL-10 levels. The DF-inoculated mice had higher levels of IL-10 compared to the DHF- and DSSinoculated mice $(p=0.007)$, with a peak at day 3 p.i ( $p$ $=0.009$ ) (Figure 3). The DF-inoculated mice also dominated in serum levels of secreted MCP-1 and showed the biggest difference compared to the DSS-inoculated mice $(p<0.0001)$, followed by DHF-inoculated mice ( $p$ $=0.011$ ) (Figure 4). MCP-1 levels peaked at day 3 p.i. whereupon it decreased $(p=<0.001)$. Inoculated virus titer showed a positive correlation to measured serum levels of RANTES in all DENV-1 inoculated mice $(p=$ $0.0036)$, and DF-inoculated mice dominated $(p=0.048)$. IL-13 and RANTES showed a dose-dependent response to inoculated virus. IL- $1 \beta$, IL-2, and TNF $\alpha$ did not show any statistically significant relationships to any of the analysed parameters (table 3).

\section{Discussion}

DENV infections are a major public health problem and constitute a real challenge due to the absence of vaccines and effective antiviral drugs. There are no non-

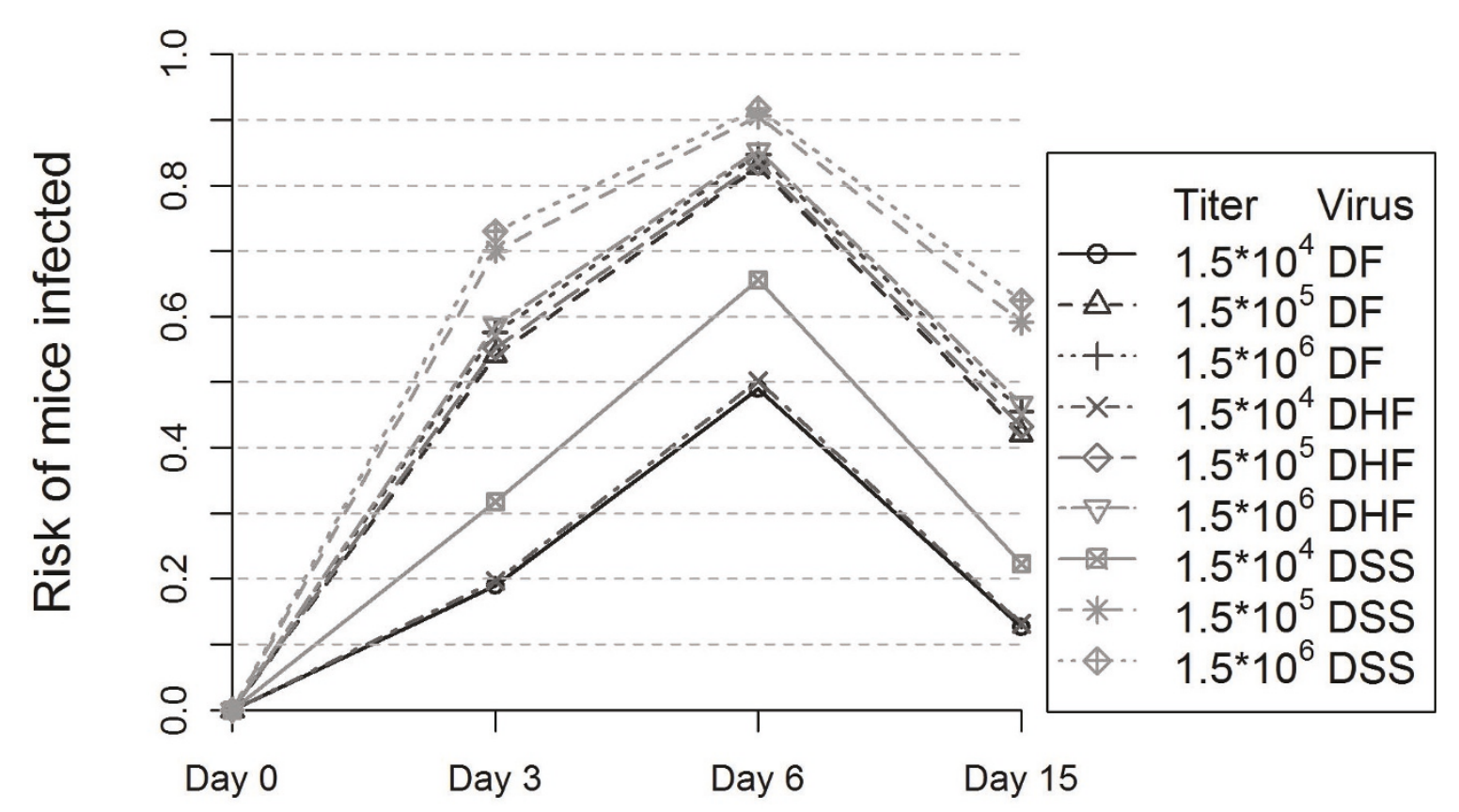

Days post-infection

Figure 1 Estimated risk of infection depending on virus concentration, day p.i. and virus isolate. 


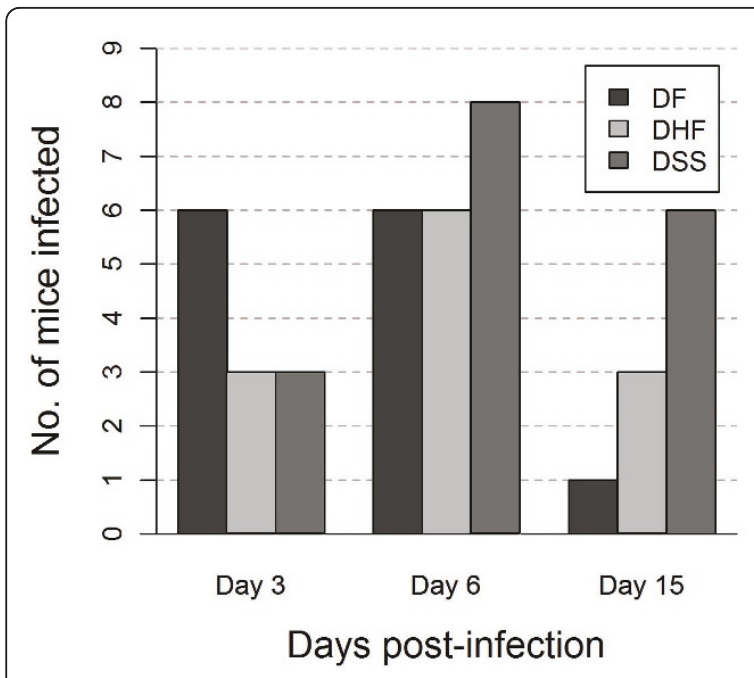

Figure 2 Persistence of infection shown as the number of mice infected out of the total mice inoculated, independent of inoculated virus concentration. Nine mice per DENV isolate and time-point in total were inoculated with the corresponding DENV isolates; DF, DHF, and DSS, respectively.

human species that naturally develops disease following DENV inoculation into peripheral sites and that resemble the clinical symptoms observed in humans [29]. The mechanisms underlying the immune responses to DENV infection are still poorly defined, and the lack of suitable animal models has hampered investigation of

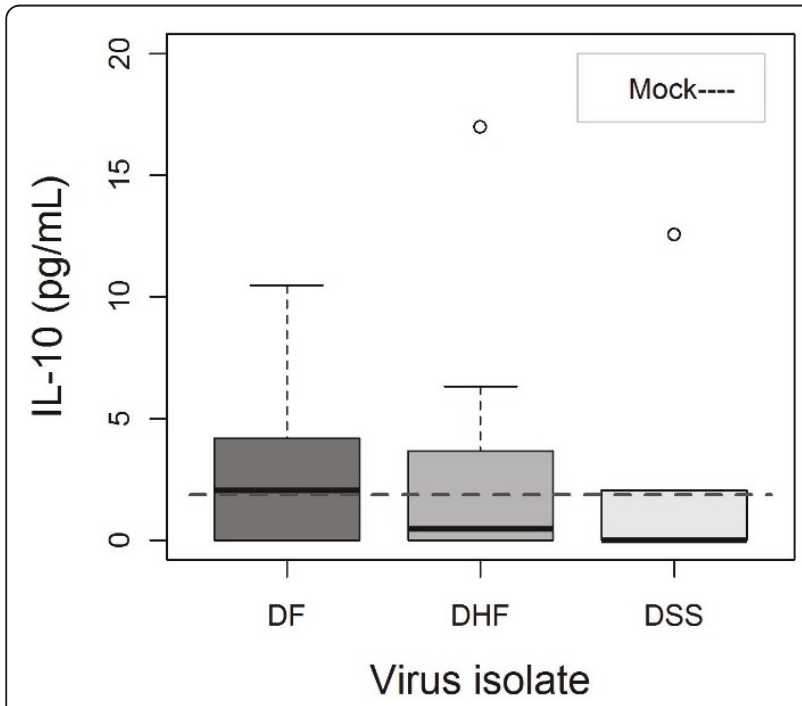

Figure 3 Box plots showing median IL-10 (solid line) by DENV1 isolates independent of inoculated virus concentration and days p.i. The line in the middle of each box represents the median; the boxes consists of $1^{\text {st }}$ and $3^{\text {rd }}$ quartile, whiskers are $1.5^{*}\left(3^{\text {rd }}\right.$ quartile- $1^{\text {st }}$ quartile). Outliers are marked with an open circle. The DF-inoculated mice had higher levels of IL-10 compared to the DHF- and DSS-inoculated mice $(p=0.007)$, with a peak at day 3 p.i $(p=0.009)$.

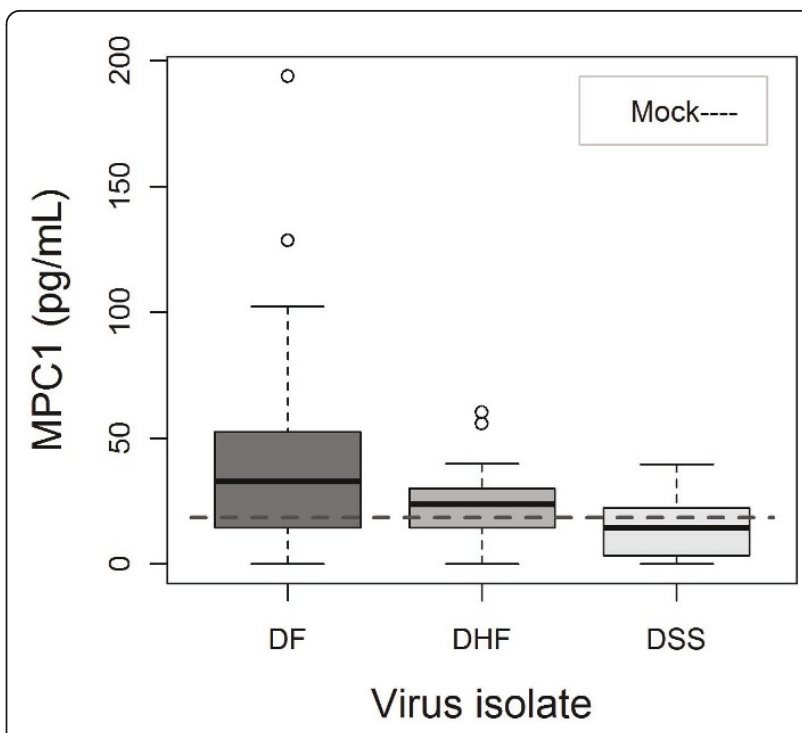

Figure 4 Box plots showing median MCP-1 (solid line) by DENV-1 isolates independent of inoculated virus concentration and days p.i. The line in the middle of each box represents the median; the boxes consists of $1^{\text {st }}$ and $3^{\text {rd }}$ quartile, whiskers are $1.5^{*}$ $\left(3^{\text {rd }}\right.$ quartile- $1^{\text {st }}$ quartile). Outliers are marked with an open circle. The DF-inoculated mice had the highest serum levels of secreted MCP-1 and showed the biggest difference compared to the DSSinoculated mice $(p<0.0001)$, followed by DHF-inoculated mice ( $p=$ 0.011). MCP-1 levels peaked at day 3 p.i. and whereupon it decreased $(p=<0.001)$.

host- and virus-specific mechanisms that control primary and sequential DENV infections.

In this study, we have compared three minimally passaged DENV-1 isolates from patients experiencing the various clinical forms of dengue disease (DF; DHF; and DSS, respectively) in vivo by infection in mouse. We show that the isolate from a DSS patient infected the largest number of BALB/c mice, with a different tropism compared to the DENV-1 isolates obtained from milder clinical cases during the same outbreak. This indicates that DENVs within the same serotype and genotype may differ enough to cause various clinical conditions in vivo. These results confirm a phenotypic distinction previously observed in vitro with the same DENV-1 isolates where the DSS isolate replicated less efficiently in mammalian Vero cells than the DF and DHF isolates, and elicited apoptosis in mosquito C6/36 cells [1].

The aim of the study was to compare wild-type features of DENV-1 isolates passaged only once in vitro and never before inoculated in mouse or cultured in mouse cells prior to direct intravenous inoculation into $\mathrm{BALB} / \mathrm{c}$ mice. BALB/c mice are known to be susceptible for DENV infection although with discrete pathological features $[11,30]$. We found that the mice did not develop dengue disease, and did not present any symptoms of the infection. However, viral RNA was detected 
Table 3 Serum cytokine levels in mice inoculated with DENV-1 isolates from patients with DF, DHF, and DSS, respectively, and independent of inoculated virus concentration

\begin{tabular}{|c|c|c|c|c|c|}
\hline Day p.i. & Cytokine & DF & DHF & DSS & Mock \\
\hline \multirow[t]{9}{*}{3} & IFN $\gamma$ & 3.10 & 3.24 & 2.08 & 0.09 \\
\hline & $\mathrm{IL}-1 \beta$ & 3.15 & 0.00 & 0.00 & 0.00 \\
\hline & IL-2 & 1.46 & 2.00 & 1.05 & 0.00 \\
\hline & IL-6 & 1.48 & 1.65 & 2.69 & 3.22 \\
\hline & IL-10 & 4.20 & 3.13 & 0.00 & 0.00 \\
\hline & IL-13 & 88.83 & 73.12 & 80.23 & 98.84 \\
\hline & MCP-1 & 71.58 & 34.25 & 21.45 & 0.00 \\
\hline & RANTES & 35.11 & 13.17 & 15.93 & 9.76 \\
\hline & TNF $\alpha$ & 6.44 & 3.97 & 3.78 & 0.00 \\
\hline \multirow[t]{9}{*}{6} & $\mathrm{IFN \gamma}$ & 2.53 & 1.65 & 1.92 & 0.00 \\
\hline & $\| \mathrm{L}-1 \beta$ & 3.15 & 0.00 & 0.00 & 0.00 \\
\hline & IL-2 & 1.46 & 1.44 & 2.21 & 2.21 \\
\hline & IL-6 & 0.36 & 0.77 & 3.10 & 2.69 \\
\hline & IL-10 & 2.05 & 0.00 & 0.00 & 0.00 \\
\hline & IL-13 & 70.84 & 62.33 & 51.19 & 121.60 \\
\hline & MCP-1 & 22.12 & 14.54 & 3.37 & 0.00 \\
\hline & RANTES & 20.47 & 14.16 & 24.46 & 10.43 \\
\hline & TNF $\alpha$ & 2.45 & 2.15 & 0.00 & 0.00 \\
\hline \multirow[t]{9}{*}{15} & $\mathrm{IFN \gamma}$ & 1.24 & 1.28 & 1.70 & 0.87 \\
\hline & $\| \mathrm{L}-1 \beta$ & 0.00 & 0.00 & 0.00 & 0.00 \\
\hline & IL-2 & 2.20 & 1.19 & 5.61 & 0.00 \\
\hline & IL-6 & 0.18 & 1.88 & 1.65 & 0.03 \\
\hline & IL-10 & 1.03 & 0.00 & 0.00 & 0.00 \\
\hline & IL-13 & 67.20 & 91.70 & 84.96 & 75.57 \\
\hline & MCP-1 & 21.53 & 14.54 & 3.37 & 0.00 \\
\hline & RANTES & 27.68 & 17.22 & 20.75 & 15.02 \\
\hline & TNF $\alpha$ & 1.73 & 0.00 & 1.48 & 0.00 \\
\hline
\end{tabular}

The cytokines were measured in $\mathrm{pg} / \mathrm{mL}$.

in various organs depending on the DENV isolate injected (table 1). We were unable to detect any systemic viremia and this suggests an absence or a very low level of virus replication in circulating peripheral blood mononuclear cells. Alternatively, the lack of detectable viraemia could indicate that the virus had already disseminated into peripheral sites, since tissue infection was measured from day 3 p.i.

In brief, viral RNA was detected by qRT-PCR in spleen, liver, lungs, kidneys, and brain. Viral RNA was found in the kidneys in only one mouse, which is in line with earlier studies [31]. Organ tropism clearly differed between the three clinical DENV-1 isolates and our findings suggest that the disease severity may correlate with high tissue viral burden, even if plasma viraemia was below the detection limit. The DENV isolate from a DSS case showed a strong preference for brain tissue, compared to the two isolates from milder dengue cases that primarily were found in other organs as lungs and liver. Neurotropism in mice has been previously suggested to be associated with DENV virulence when comparing DENV-3 isolated from Brazilian patients [32].

Breakdown of the blood-brain barrier in DENV infected mice have been described before, and were shown to be dose-dependent [33]. Genomic sequencing performed previously [1] identified a unique amino acid substitution $\mathrm{L} \rightarrow \mathrm{F} 476$ in the $\mathrm{E}$ protein of the DSS isolate compared to the DF and DHF isolates, that may explain the neurotropic character of the DSS-isolate. The NS1 gene of the DSS isolate harboured another unique amino acid substitution, $\mathrm{K} \rightarrow \mathrm{R} 115$ in comparison to the two other DENV-1 isolates. The soluble form of NS1 (sNS1) is a dominant target of humoral immunity and activates complement components in normal human serum, and is proposed to play a significant role in the pathogenesis of disease [25,34]. The NS1 glycoprotein is glycosylated at two sites, N130 and N270, and glycosylation of both residues has been suggested to be required for neurovirulence in mice [35]. It is remains to be elucidated whether the possible breakdown of the bloodbrain barrier and neurotropic character of the DSS isolate has any correlation to the disruption of the endothelium observed in humans suffering from vascular leakage. The three remaining amino acid substitutions unique for the DSS isolate were located in the NS protein $3(\mathrm{~S} \rightarrow \mathrm{P} 118)$, and $5(\mathrm{~T} \rightarrow \mathrm{I} 49$, and $\mathrm{S} \rightarrow \mathrm{N} 830)$. These differences could potentially alter the enzymatic activity of these proteins.

The DSS isolate seemed to persist longer in vivo since viral RNA was detected in a majority of mice on day 15 p.i. compared to the DF and DHF infected mice, and thereby exhibited infection kinetics differing from the two other DENV-1 isolates (DF and DHF) where viral RNA decreased after day 6 p.i. Thus, the DSS isolate could be regarded as more virulent than the two other DENV-1 strains, since a majority of the DSS-inoculated mice had detectable levels of viral RNA, infection was also less affected by the dose of inoculums and persisted longer in a majority of mice (Figure 1). The wild-type DENVs analyzed here could provide evidence for differences in virus replication, which in turn could influence the clinical outcomes of the infection, and eventually partitally explain some differences in virulence observed in humans.

These results confirm the in vitro findings of the same DENV-1 isolates, that showed that the strains derived from a DSS patient can be distinguished based on phenotypic characteristics from the isolates derived from a DF and DHF case [1]. We have previously shown that the DSS virus isolate exhibit much slower replication kinetics in mammalian Vero cells and an apoptotic 
response in mosquito cells, and we observed in this study that the DSS virus isolate persist longer in vivo with extensive neuroinvasion.

Cytokines involved in inflammation were quantified in serum collected from inoculated mice and compared in regard to DENV-1 isolate, virus titer inoculated, and time-point p.i. Overall, the levels of the proinflammatory cytokines (IFN $\gamma$, IL-1 $\beta$, IL-2, IL-6, IL-10, IL-13, MCP-1, RANTES, and TNF $\alpha$ ) quantified were low, which is most probably due to fact that BALB/c mice is poorly susceptible to wild-type DENVs. The mice did not develop clinical apparent disease, and showed only discrete lesions in internal organs. Common for several cytokines, however, was a dose-dependent response to inoculated virus (IFN $\gamma$, IL-6, IL-13, and RANTES). The highest levels of measured cytokines were also in general at the beginning of the experimental period (IFN $\gamma$, IL10 , and MCP-1 peaked at day 3 p.i.). A curious finding was that DF-inoculated mice had higher detectable serum levels of IL-10, MCP-1, and RANTES, which have been seen to be increased in patients with severe DHF and DSS [36-42]. The preference for the brain of the DHF-, and most notably the DSS-infected mice, could imply locally high levels of proinflammatory cytokines that do not circulate systemically. This could explain why the IL-10, MCP-1, and RANTES levels dominated in the DF-inoculated mice, since the thoracic and abdominal organs were the primary target for DF isolate. Cytokines like IL-1 $\beta$, IL-2, and TNF $\alpha$ did not show any differences compared to mock-infected controls and this could be due to the transient nature of many cytokines as well as the limited susceptibility of DENVs in mice.

\section{Conclusions}

We have previously shown in vitro that the isolate from a DSS case could be distinguished based on replication kinetics and apoptosis from isolates originating in milder cases. In this study we have extended the characterization of those clinical DENV-1 isolates derived from patients exhibiting the various forms of dengue illness. Despite their close relatedness, the DENV-1 isolate from a DSS case was phenotypically different from the other two isolates by significant brain invasiveness and a higher infectivity in BALB/c mice. The isolates from a DF and DHF case infected primarily lungs and liver, and to a limited extent brain and infection declined faster after day 6 p.i than in DSS-infected mice.

This is the first time different clinical South-East Asian DENV isolates have been directly compared for the in vivo characteristics in BALB/c mice. To define key elements involved in the virulence of these characterized DENV-1 phenotypes reverse genetics systems are needed. Additional low-passage strains of the different
DENV serotypes of each clinical forms of dengue are required to fully decipher the complex mechanisms governing DENV pathogenesis.

\section{Materials and methods \\ Virus}

Three dengue serotype-1 (DENV-1) virus strains isolated from patient sera during a DENV outbreak in the Kampong Cham province, Eastern Cambodia, in 2007, were used. The isolates were obtained from patients experiencing the three distinct clinical forms of dengue disease: DF, DHF and DSS, according to the WHO classification [43]. Patient information regarding the analysed clinical DENV isolates is presented in previous work by Tuiskunen, A. et al., 2011. Blood samples were collected between day 2 and 6 after onset of disease and the serotypes were determined at the Institute Pasteur in Cambodia (IPC) by nested reverse transcriptase-polymerase chain reaction, according to Lanciotti procedure modified by Reynes et al. [44]. The virus isolates were obtained during the DENFRAME study, which has been approved by the Cambodian National Ethics Committee and patient's enrolment was subject to obtaining a written consent signed by the patients, or the under 16 year old patient's legal representatives.

Each DENV was isolated in A. albopictus mosquito cell line C6/36 (CRL 1660, ATCC), and thereafter propagated by one passage. The C6/36 cells were maintained in Leibovitz-15 medium, supplemented with $2 \%$ tryptose phosphate broth, and 5\% fetal calf serum (Invitrogen, Stockholm, Sweden) and maintained at $28^{\circ} \mathrm{C}$ for 6 days. Cell culture supernatant was centrifuged for 10 min at $15000 \mathrm{rpm}$, titrated as previously described [1] and stored at $-80^{\circ} \mathrm{C}$ in a solution of sucrose $[1.5 \mathrm{M}]$ and hepes $[1 \mathrm{M}]$. Virus had not undergone any previous passage in mouse.

\section{Animals}

The animal experimental procedures were approved by the Committee for Laboratory Animal Science of the Swedish Board of Agriculture (ethical permit ID no: 339/07).

Female BALB/c mice (Nova, Sollentuna, Sweden) age 6 weeks weighing $20 \mathrm{~g}$ were maintained on a standard laboratory diet with water ad libitum and housed under specific pathogen-free conditions at the animal facility of the Swedish Institute for Communicable Disease Control (SMI) in Stockholm, Sweden. Mice subjected to the same treatment were kept in groups of 4-5 mice per cage and handled according to the international guidelines for experimentation on animals. DENV-1 infected mice were housed in a biological safety level (BSL) 3 isolator, and negative control mice were housed in a BSL 2 facility. 
Table 4 Schematic overview of the experimental design

\begin{tabular}{|c|c|c|c|c|c|c|c|c|c|c|}
\hline \multirow{2}{*}{$\begin{array}{r}\text { DENV-1 isolate } \\
\text { Titer PFU/mL }\end{array}$} & \multicolumn{3}{|c|}{ DF } & \multicolumn{3}{|c|}{ DHF } & \multicolumn{3}{|c|}{ DSS } & \multirow{2}{*}{$\begin{array}{l}\text { NC } \\
\text { N.A }\end{array}$} \\
\hline & $1.5 \times 10^{4}$ & $1.5 \times 10^{5}$ & $1.5 \times 10^{6}$ & $1.5 \times 10^{4}$ & $1.5 \times 10^{5}$ & $1.5 \times 10^{6}$ & $1.5 \times 10^{4}$ & $1.5 \times 10^{5}$ & $1.5 \times 10^{6}$ & \\
\hline Day 3 p.i & 3 & 3 & 3 & 3 & 3 & 3 & 3 & 3 & 3 & 9 \\
\hline Day 6 p.i & 3 & 3 & 3 & 3 & 3 & 3 & 3 & 3 & 3 & 9 \\
\hline Day 15 p.i & 3 & 3 & 3 & 3 & 3 & 3 & 3 & 3 & 3 & 9 \\
\hline Total no. of mice & & 27 & & & 27 & & & 27 & & 18 \\
\hline
\end{tabular}

Mice were divided in groups of three depending on DENV-1 isolate for inoculation; titer of inoculum; and time of euthanasia. There were in total 99 mice included in the study, whereof 81 were inoculated with infectious DENV-1.

N.A. = not applicable

\section{DENV-1 infection in mice}

There were 99 mice in total divided into four groups with 27 mice each according to the 3 DENV-1 isolates and 18 mock infected negative controls (NC). Each group of 27 mice were subdivided into three groups of nine mice that were injected intravenously (i.v.) in the tail vein with a single dose $\left(1.5 \times 10^{4} \mathrm{PFU} / \mathrm{mL} ; 1.5 \times\right.$ $10^{5} \mathrm{PFU} / \mathrm{mL}$; or $1.5 \times 10^{6} \mathrm{PFU} / \mathrm{mL}$, respectively) of infective DENV-1 without adjuvant. Similarly, the NC group of 18 mice received $150 \mathrm{uL}$ of Leibovitz-15 medium supplemented with sucrose $[1.5 \mathrm{M}]$ and hepes [1 M] (table 4). The mice were monitored daily for clinical signs.

Blood that was obtained via a cardiac puncture of mice immediately after euthanasia by $4.5-4.8 \%$ isoflurane gas inhalation on day 3; 6, and 15 p.i. All mice were immediately splenectomised after euthanasia and thereafter stored at $-80^{\circ} \mathrm{C}$.

\section{Detection of DENV RNA in BALB/c mice}

To assess viral burden in tissues of infected mice, organs such as liver, heart, spleen, lungs, brain, and kidneys were harvested, weighed, and homogenized using Stainless Steel Beads (5.0 mm diameter) with a TissueLyser apparatus (Qiagen, Hilden, Germany). Total RNA from tissues was extracted immediately after dissection using the RNeasy Mini kit according to the manufacturer's instructions (Qiagen).

Mouse sera were obtained by centrifugation of whole blood for 3 minutes at $2000 \mathrm{~g}$ in microtainer EDTA tubes (Becton, Dickinson and Company, Temse, Belgium). Viral RNA from thawed aliquots of serum (140 $\mathrm{uL}$ ) was extracted using QIAamp Viral RNA Mini kit (Qiagen). RNA samples, eluted in RNAse-free water, were stored at $-80^{\circ} \mathrm{C}$. Quantitative measurements of total viral RNA were performed as previously described [45].

IFA for virus-specific antibody detection

An in-house immunofluorescence assay (IFA) was used as previously described [46] essentially to verify that all DENV-1 infected mice had raised a DENV specific antibody response.

\section{Cytokine dosage}

Cytokine levels in mouse sera collected from all inoculated mice, including mock infected, were measured as duplicates with Milliplex Mouse Cytokine/Chemokine Panel (Millipore) in a Luminex 100 (Luminex, Bio-Rad, Sweden). The following cytokines and chemokines were analysed according to the manufacturer's instructions: IFN $\gamma$, IL-1 $\beta$, IL-2, IL-6, IL-10, IL-13, MCP-1, RANTES, and TNF $\alpha$.

\section{Statistical Analysis}

To test the effect of virus, titer and days p.i. on the risk of DENV infection in mouse organs binomial regression with a log link was used. Parametric bootstrap with 10.000 replicates was drawn from an assumed binomial probability model. The effect of virus, titer and day between smaller and larger models were then evaluated by comparing the deviance, extracted from the replicates. The largest model, within each analysis of the infection risk in an organ, included the main effects virus, titer and day plus the interactions virus by titer, virus by day and titer by day.

Median regression was used to evaluate the relationship between virus isolate, day, and titer on the different cytokines. As most cytokines displayed highly right skewed distributions in some virus isolates the use of median estimates yielded more robust results than with the use of mean based methods. Day and titer were analyzed separately with two main models per cytokine: Cytokine $=$ group + day + group by day interaction; and Cytokine $=$ group + titer + group by titer interaction. Pvalues $<0.05$ were used as statistically significant results. All analyses and graphs were performed using the statistical software R (version 2.13.1, 2011).

\section{Acknowledgements}

We thank the animal technicians Christel Werner and Olov Rosendahl at the animal facility of the Swedish Institute for Communicable Disease Control, and Dr Mikael Andersson for invaluable help with the statistical analysis.

\section{Author details}

${ }^{1}$ Department of Microbiology, Tumor and Cell Biology, Karolinska Institutet, Stockholm, Sweden. 'Swedish Institute for Communicable Disease Control, Solna, Sweden. ${ }^{3}$ Virology Department, French Army Forces Biomedical 
Institute (IRBA), Marseille, France. ${ }^{4}$ Virology Unit, Institut Pasteur in Cambodia, Phnom Penh, Cambodia.

\section{Authors' contributions}

Conceived and designed the experiments: AT, ILG. Performed the experiments: AT, MW. Analyzed the data: AT, ÅL, ILG. Wrote the paper: AT. Reviewed the paper: PB, ILG, ÅL. All authors read and approved the final manuscript.

\section{Competing interests}

The authors declare that they have no competing interests.

Received: 28 July 2011 Accepted: 11 August 2011

Published: 11 August 2011

\section{References}

1. Tuiskunen A, Monteil V, Plumet S, Boubis L, Wahlstrom M, Duong V, Buchy P, Lundkvist A, Tolou H, Leparc-Goffart I: Phenotypic and genotypic characterization of dengue virus isolates differentiates dengue fever and dengue hemorrhagic fever from dengue shock syndrome. Arch Virol 2011.

2. Rice $C M$, et al: Nucleotide sequence of yellow fever virus: implications for flavivirus gene expression and evolution. Science 1985, 229(4715):726-33.

3. Chambers $\mathrm{TJ}$, et al: Flavivirus genome organization, expression, and replication. Annu Rev Microbiol 1990, 44:649-88.

4. Halstead SB: Pathogenesis of dengue: challenges to molecular biology. Science 1988, 239(4839):476-81.

5. WHO: Dengue: guidelines for diagnosis, treatment, prevention and control - New edition. World Health Organization; 2009.

6. Gibbons RV, Vaughn DW: Dengue: an escalating problem. BMJ 2002, 324(7353):1563-6.

7. Sakuntabhai $A$, et al: $A$ variant in the $C D 209$ promoter is associated with severity of dengue disease. Nat Genet 2005, 37(5):507-13.

8. An J, et al: Development of a novel mouse model for dengue virus infection. Virology 1999, 263(1):70-7.

9. Blaney JE Jr, et al: Genetic basis of attenuation of dengue virus type 4 small plaque mutants with restricted replication in suckling mice and in SCID mice transplanted with human liver cells. Virology 2002, 300(1):125-39.

10. Lin $Y L$, et al: Study of Dengue virus infection in SCID mice engrafted with human K562 cells. J Virol 1998, 72(12):9729-37.

11. Atrasheuskaya A, et al: Anti-TNF antibody treatment reduces mortality in experimental dengue virus infection. FEMS Immunol Med Microbiol 2003, 35(1):33-42.

12. Johnson AJ, Roehrig JT: New mouse model for dengue virus vaccine testing. J Virol 1999, 73(1):783-6.

13. Lee $E$, et al: Virulence attenuation of Dengue virus due to augmented glycosaminoglycan-binding affinity and restriction in extraneural dissemination. J Gen Virol 2006, 87(Pt 10):2791-801.

14. Chen ST, et al: CLEC5A is critical for dengue-virus-induced lethal disease. Nature 2008, 453(7195):672-6.

15. Prestwood TR, et al: A mouse-passaged dengue virus strain with reduced affinity for heparan sulfate causes severe disease in mice by establishing increased systemic viral loads. J Virol 2008, 82(17):8411-21.

16. Shresta $S$, et al: Murine model for dengue virus-induced lethal disease with increased vascular permeability. J Virol 2006, 80(20):10208-17.

17. Barreto DF, et al: Histopathological and ultrastructural aspects of mice lungs experimentally infected with dengue virus serotype 2 . Mem Inst Oswaldo Cruz 2007, 102(2):175-82.

18. Paes MV, et al: Liver injury and viremia in mice infected with dengue-2 virus. Virology 2005, 338(2):236-46

19. Huang KJ, et al: Manifestation of thrombocytopenia in dengue-2-virusinfected mice. J Gen Virol 2000, 81(Pt 9):2177-82.

20. Chen $\mathrm{HC}$, et al: Both virus and tumor necrosis factor alpha are critical for endothelium damage in a mouse model of dengue virus-induced hemorrhage. J Virol 2007, 81(11):5518-26.

21. Zulueta A, et al: Amino acid changes in the recombinant Dengue 3 Envelope domain III determine its antigenicity and immunogenicity in mice. Virus Res 2006, 121(1):65-73.

22. Navarro-Sanchez E, Despres P, Cedillo-Barron L: Innate immune responses to dengue virus. Arch Med Res 2005, 36(5):425-35.
23. Modis $Y$, et al: Structure of the dengue virus envelope protein after membrane fusion. Nature 2004, 427(6972):313-9.

24. Schlesinger JJ, Brandriss MW, Walsh EE: Protection of mice against dengue 2 virus encephalitis by immunization with the dengue 2 virus nonstructural glycoprotein NS1. J Gen Virol 1987, 68(Pt 3):853-7.

25. Avirutnan $P$, et al: Vascular leakage in severe dengue virus infections: a potential role for the nonstructural viral protein NS1 and complement. J Infect Dis 2006, 193(8):1078-88.

26. Chambers TJ, Grakoui A, Rice CM: Processing of the yellow fever virus nonstructural polyprotein: a catalytically active NS3 proteinase domain and NS2B are required for cleavages at dibasic sites. J Virol 1991, 65(11):6042-50.

27. Gorbalenya $A E$, et al: N-terminal domains of putative helicases of flaviand pestiviruses may be serine proteases. Nucleic Acids Res 1989, 17(10):3889-97.

28. Bazan JF, Fletterick RJ: Detection of a trypsin-like serine protease domain in flaviviruses and pestiviruses. Virology 1989, 171(2):637-9.

29. Bente DA, Rico-Hesse R: Models of dengue virus infection. Drug Discov Today Dis Models 2006, 3(1):97-103.

30. Gil L, et al: The cellular immune response plays an important role in protecting against dengue virus in the mouse encephalitis model. Viral Immunol 2009, 22(1):23-30.

31. Mota J, Rico-Hesse R: Humanized mice show clinical signs of dengue fever according to infecting virus genotype. J Virol 2009, 83(17):8638-45.

32. Ferreira GP, et al: Dengue virus 3 clinical isolates show different patterns of virulence in experimental mice infection. Microbes Infect 2010, 12(7):546-54.

33. Chaturvedi UC, et al: Breakdown of the blood-brain barrier during dengue virus infection of mice. J Gen Virol 1991, 72(Pt 4):859-66.

34. Lin CF, et al: Autoimmune pathogenesis in dengue virus infection. Viral Immunol 2006, 19(2):127-32.

35. Crabtree MB, Kinney RM, Miller BR: Deglycosylation of the NS1 protein of dengue 2 virus, strain 16681: construction and characterization of mutant viruses. Arch Virol 2005, 150(4):771-86

36. Chaturvedi UC, et al: Cytokine cascade in dengue hemorrhagic fever: implications for pathogenesis. FEMS Immunol Med Microbiol 2000, 28(3):183-8.

37. Chaturvedi UC, Raghupathy R, Pacsa AS, Elbishbishi EA, Agarwal R, Nagar R, Misra A, Kapoor S, mathur A, Khan MAY, Azizieh F: Shift from a Th1-type response to Th2-type in dengue hemorrhagic fever. Curr Sci 1999, 76:63-69.

38. Green $S$, et al: Elevated plasma interleukin-10 levels in acute dengue correlate with disease severity. J Med Virol 1999, 59(3):329-34.

39. Azeredo EL, et al: Characterisation of lymphocyte response and cytokine patterns in patients with dengue fever. Immunobiology 2001. 204(4):494-507.

40. Chakravarti A, Kumaria R: Circulating levels of tumour necrosis factoralpha \& interferon-gamma in patients with dengue \& dengue haemorrhagic fever during an outbreak. Indian J Med Res 2006, 123(1):25-30

41. Yang KD, et al: A model to study cytokine profiles in primary and heterologously secondary Dengue-2 virus infections. Acta Virol 1995, 39(1):19-21.

42. Yang KD, Wang $\mathrm{CL}$, Shaio MF: Production of cytokines and platelet activating factor in secondary dengue virus infections. J Infect Dis 1995, 172(2):604-5.

43. WHO: Dengue haemorrhagic fever. Diagnosis, treatment, prevention and control. Geneva: WHO; 2 1997, 12-23.

44. Reynes JM, et al: Improved molecular detection of dengue virus serotype 1 variants. J Clin Microbiol 2003, 41(8):3864-7.

45. Leparc-Goffart I, et al: Development and validation of real-time one-step reverse transcription-PCR for the detection and typing of dengue viruses. J Clin Virol 2009, 45(1):61-6.

46. Vene S, Mangiafico J, Niklasson B: Indirect immunofluorescence for serological diagnosis of dengue virus infections in Swedish patients. Clin Diagn Virol 1995, 4(1):43-50.

doi:10.1186/1743-422X-8-398

Cite this article as: Tuiskunen et al:: Phenotypic characterization of patient dengue virus isolates in $B A L B / c$ mice differentiates dengue fever and dengue hemorrhagic fever from dengue shock syndrome. Virology Journal 2011 8:398. 